05

\title{
Энергетические характеристики малых металлических кластеров, содержащих вакансию
}

\author{
() В.И. Рева, В.В. Погосов
}

Запорожский национальный технический университет, 69063 Запорожье, Украина

e-mail: vpogosov@zntu.edu.ua

(Поступило в Редакцию 21 марта 2017 г.)

В рамках модели стабильного желе выполнены самосогласованные вычисления пространственных распределений электронов, потенциалов, энергий диссоциации, когезии, образования вакансии, прилипания электронов и потенциала ионизации сплошных кластеров $\mathrm{Al}_{N}, \mathrm{Na}_{N}(N \leq 254)$ и кластеров, содержащих вакансию $(N \geq 12)$. Обсужден вклад моновакансии в энергетику кластера, размерные зависимости характеристик и их асимптотик. Расчет выполнен на кластере СКИТ-3 Института Кибернетики им. В.М. Глушкова НАН Украины (Rpeak $=7.4$ Tflops $)$.

DOI: 10.21883/JTF.2018.02.45405.2258

\section{Введение}

Одна из точек зрения на плавление твердых тел [1] предполагает скачкообразное увеличение концентрации дырок-вакансий в тройной точке, а также уменьшение энергии образования вакансий с ростом их концентрации. В точке плавления концентрация вакансий в металлах составляет доли процента. Несмотря на столь малые концентрации, вакансии оказывают большое влияние на свойства твердых тел.

Равновесная концентрация вакансий вычисляется при наличии энергии образования вакансии, которая может быть извлечена из спектра аннигиляции, локализованных в вакансии позитронов. При такой процедуре концентрация вакансий остается свободным параметром.

Первоначально в экспериментах установлено, что температура плавления кластеров на подложке и свободных кластеров уменьшается с уменьшением их размеров. Интерпретации и моделированию этого мезоскопического явления посвящено значительное число работ [2-7].

Современные масс-спектрометрические и калориметрические методы, позволяющие детально исследовать процесс предплавления и плавления металлических кластеров, состоящих из счетного числа атомов [8-11], показали, что температура плавления характеризуется осцилляционной размерной зависимостью, а также имеет размерные аномалии (например, для $\mathrm{Al}$ ), плохо описываемые в простых моделях $[8,10]$. Также в процессе плавления диффузия поверхностных вакансий в объем более выгодна для кластеров с незаполненными электронными оболочками, чем для кластеров с магическим числом атомов [9]. Эти факты стимулируют повышенный интерес к рассмотрению фазового перехода из твердого в жидкое состояние, как к конфигурационному возбуждению дырок-вакансий в кластерах.

Популярна точка зрения [12-14] с позиции термодинамики: вблизи температуры плавления энергия образования вакансий тем меньше, чем меньше кластер, а концентрация вакансий не зависит от его размера. Однако термодинамика не дает указаний на механизм образования вакансий, и вопрос о размерной зависимости энергии образования вакансий, их концентрации и связи вакансий с процессом плавления остается открытым.

Несмотря на то что энергетические характеристики сплошных металлических кластеров рассчитывались неоднократно в различных моделях ([15-17] и ссылки в них), самосогласованные вычисления энергии образования вакансии в кластерах и влияние на нее квантования электронного спектра до сих пор не выполнялись. Помимо этой задачи, одной из актуальных задач, которые можно сформулировать в связи с плавлением малоразмерных агрегатов, является исследование размерного поведения потенциала ионизации кластеров, содержащих моновакансию. Полученные зависимости могут быть полезными при проведении анализа результатов фотоионизационных экспериментов, а также для определения размерной зависимости концентрации вакансий, в том числе вблизи температуры плавления.

Целью настоящей работы является расчет методом Кона-Шема в модели стабильного желе целого набора энергетических характеристик кластеров, содержащих вакансию, что позволило установить, в частности, как размерное поведение энергии образования вакансии, так и ее зависимость от способа ее создания.

\section{Основные соотношения}

Представим металлический кластер в виде сферы. Будем сравнивать характеристики сфер с одинаковым числом атомов $N$ при нулевой температуре. При этом радиусы сплошных сфер $R_{N}$ и сфер с моновакансией в центре $R_{N, v}$ отличаются друг от друга:

$$
\begin{gathered}
R_{N}=N^{1 / 3} r_{0}, \\
R_{N, v}=(N+1)^{1 / 3} r_{0},
\end{gathered}
$$

где $r_{0}$ радиус ячейки Вигнера-Зейтца, приходящийся на один атом. 

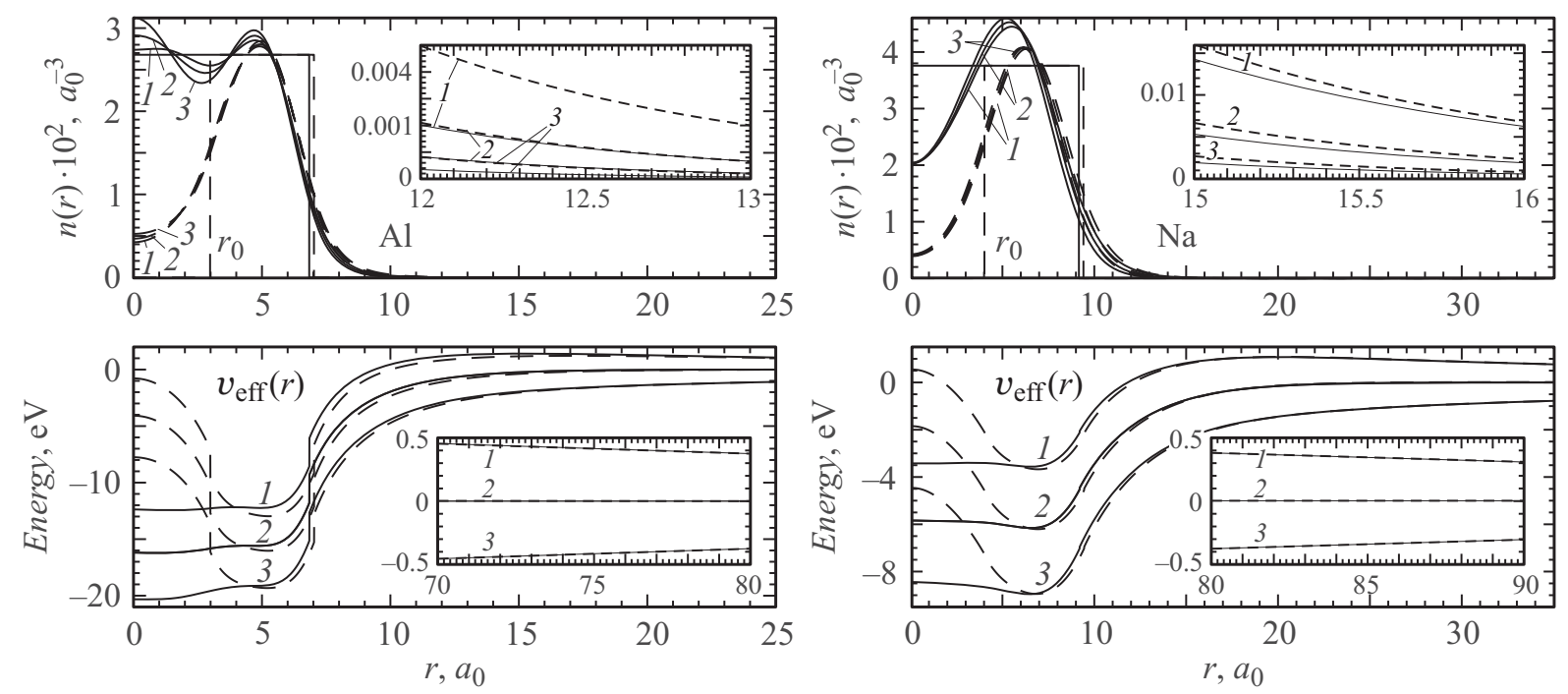

Рис. 1. Самосогласованные профили электронного распределения $n(r)$ и эффективного потенциала $v_{\mathrm{eff}}(r)$ для заряженного и нейтрального сплошного кластера (сплошные линии) и кластера с вакансией в центре (штриховые линии), содержащих одинаковое число атомов $N=12 ; 1-Q=-e, 2-0,3-+e ; a_{0}-$ боровский радиус.

В модели стабильного желе моновакансию представляют в виде сферической нейтральной дырки радиуса $r_{0}$ в однородном положительно заряженном фоне, создаваемом ионами. Распределение ионного заряда кластера с вакансией запишем, используя единичные ступенчатые $\theta$-функции:

$$
\rho_{v}(r)=\bar{n} \theta\left(r-r_{0}\right) \theta\left(R_{V, v}-r\right),
$$

где $\bar{n}=3 Z /\left(4 \pi r_{0}^{3}\right)-$ концентрация однородного электронного газа, $Z$ - валентность металла. Для сплошного (бездефектного) кластера $\rho(r)=\bar{n} \theta\left(R_{N}-r\right)$.

Полную энергию металлической сферы с вакансией в центре представим в виде функционала электронной концентрации $(e-$ элементарный положительный заряд)

$$
\begin{aligned}
& E_{N, v}=T_{s, v}+\frac{e}{2} \int d^{3} r \phi_{v}(r)\left[n_{v}(r)-\rho_{v}(r)\right] \\
& +\int d^{3} r n_{v}(r) \varepsilon_{x c, v}(r)-\Delta \bar{\varepsilon} \int d^{3} r \rho_{v}(r)+\langle\delta v\rangle_{W S} \\
& \times \int d^{3} r \theta\left(r-r_{0}\right) \theta\left(R_{N, v}-r\right) n_{v}(r),
\end{aligned}
$$

где

$$
T_{s, v}=\sum_{i=1}^{N_{e}} \varepsilon_{i, u}-\int d^{3} r n_{v}(r) v_{\mathrm{eff}, v}(r)
$$

- кинетическая энергия невзаимодействующих электронов числом $N_{e}=Z N$. Последние два члена в (3) учитывают структуру ионной подсистемы, а также электрон-ионное взаимодействие в виде псевдопотенциала Ашкрофта (см. детали в $[18,19])$.

В версии Кона-Шема профиль электронного распределения $n_{v}(r)$ кластера с вакансией, выражается посредством одноэлектронных волновых функций

$$
n_{v}(r)=\sum_{i=1}^{N}\left|\psi_{i, v}(r)\right|^{2}
$$

Волновые функции $\psi_{i, v}$ и собственные значения энергий $\varepsilon_{i, v}$ находятся решением системы волновых уравнений

$$
-\frac{\hbar^{2}}{2 m} \nabla^{2} \psi_{i, v}(r)+v_{\mathrm{eff}, v}(r) \psi_{i, v}(r)=s_{i, v} \psi_{i, v}(r)
$$

с эффективным одноэлектронным потенциалом

$v_{\text {eff }}(r)=e \phi_{v}(r)+v_{x c, v}(r)+\langle\delta v\rangle_{W S} \theta\left(r-r_{0}\right) \theta\left(R_{N, v}-r\right)$,

включающим электростатический $\phi_{v}(r)$ и обменнокорреляционный потенциал $v_{x c, v}\left[n_{v}(r)\right]$ в LDA. Отсчет энергии выбран от вакуумного уровня, т.е. от энергии электрона с нулевой кинетической энергией, расположенного вдали от образца (при $r \gg R_{N, v}$ ), где нет сторонних зарядов.

Пространственное распределение электростатического потенциала $\phi_{v}(r)$ находится решением уравнения Пуассона

$$
\nabla^{2} \phi_{v}(r)=-4 \pi e\left[n_{v}(r)-\rho_{v}(r)\right]
$$

при фиксированном условии

$$
\int_{0}^{\infty} d r 4 \pi r^{2}\left[\rho_{v}(r)-n_{v}(r)\right]=Q / e,
$$

где $Q$ - суммарный заряд кластера.

Симметрия задачи позволяет разделить переменные в (6). В этом случае одноэлектронные волновые функции и энергии характеризуются радиальным $n_{r}$ и орбитальным $l$ квантовыми числами. Шаг дискретизации по $r$ 


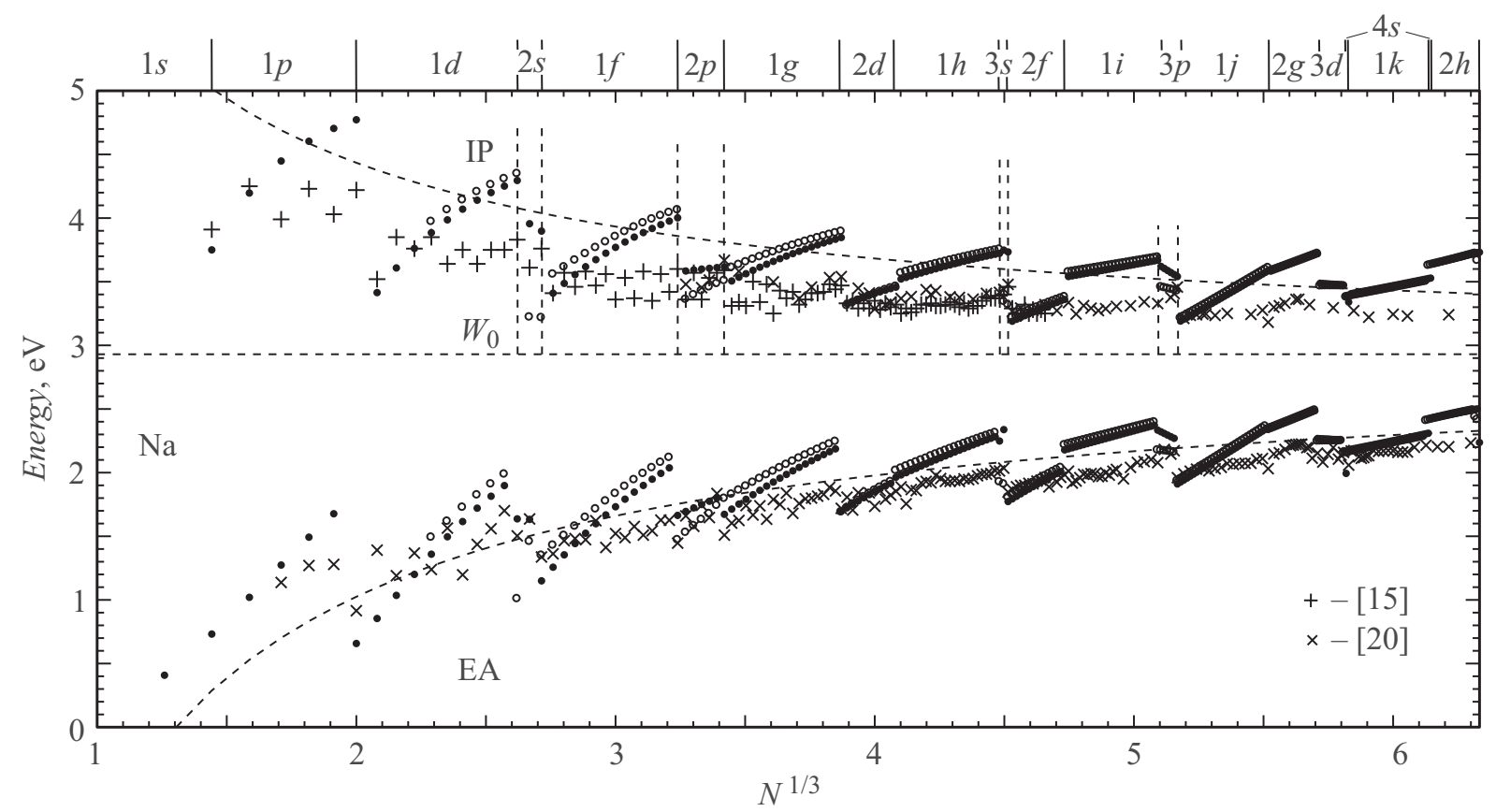

Рис. 2. Потенциал ионизации IP и энергия прилипания ЕА, вычисленные непосредственно по формулам (10) для сплошных кластеров (точки) и кластеров с моновакансией (пустые кружки); экспериментальные значения (кресты) для Na [15,20]. Штрихпунктирная линия - асимптотика (12).

составлял примерно $0.002 a_{0}, a_{0}-$ боровский радиус. Численное решение системы волновых уравнений и уравнения Пуассона проводилось методом Нумерова.

На рис. 1 приведены профили электронного распределения и эффективного потенциала для сплошного кластера и кластера с вакансией в центре, содержащих одинаковое число атомов $N=12$. На рисунке обозначены радиусы вакансии и кластеров $\left(Z=3\right.$ и $r_{0}=2.99 a_{0}$ для $\mathrm{Al} ; z=1$ и $r_{0}=3.99 a_{0}$ для $\left.\mathrm{Na}\right)$. Радиусы их отличаются в соответствии с определением (1). На вставках проиллюстрирован ход электронных профилей и потенциала вдали от кластеров. Несмотря на то что электронные распределения быстро убывают, хвосты потенциалов простираются далеко (расчет велся примерно до $\left.r=R_{N}+900 a_{0}\right)$. Для заряженных кластеров электростатический потенциал асимптотически за поверхностью убывает как $Q / r$. Для больших кластеров пространственный профиль становится похожим на профиль вблизи поверхности полубесконечного металла, содержащий большое число фриделевских осцилляций. Найденные профили позволяют вычислить полную энергию кластера (3).

\section{Потенциал ионизации и энергия прилипания электронов}

Кластеры атомов обладают структурной периодичностью, которая носит не трансляционный характер, а имеет свойство „сферической периодичности“. Эта периодичность обусловлена сферическими слоями атомов (атомными оболочками или координационными сферами). По аналогии для кластеров с вакансиями мы можем ввести минимальное число $N=12$, соответствующее сферичности задачи.

Другая особенность, присущая только металлическим кластерам, обусловлена мерой заполнения электронных оболочек, по мере увеличения количества атомов. Кластеры с заполненными электронными оболочками обладают повышенной стабильностью по сравнению с кластерами других размеров, оболочки которых частично заполнены. В пределе $N \rightarrow \infty$ разность между магическими и немагическими кластерами исчезает.

По определению, потенциал ионизации $(I P)$ и энергия прилипания электронов $(E A)$ определяются разностями полных энергий:

$$
\begin{gathered}
I P_{N, v}=E_{N, v}^{N_{e}-1}-E_{N, v}^{N_{e}} \\
E A_{N, v}=E_{N, v}^{N_{e}}-E_{N, v}^{N_{e}+1},
\end{gathered}
$$

где $E_{N, v}^{N_{e}-1} / E_{N, v}^{N_{e}+1}$ - энергии сферы радиусом $R_{N, v}$ с избыточным зарядом $Q=+e /-e, E_{N, v}^{N_{e}}-$ энергия нейтральной сферы $(Q=0)$.

На рис. 2 и 3 приведены результаты вычислений IP и $E A$. Литеры $s, p, d, f, g, h, i, j, k, l, m, n$, $o, p$ соответствуют орбитальным числам $l=0, \ldots, 13$. Картину для всего диапазона $N$ на рис. 2 мы привели только для Na. Там же можно проследить разницу между сплошными кластерами и дефектными. С ростом $N$, начиная с 12, эта разница может составлять $0.1-0.5 \mathrm{eV}$ (для $\mathrm{Al}$ - примерно вдвое больше). Максимальное 


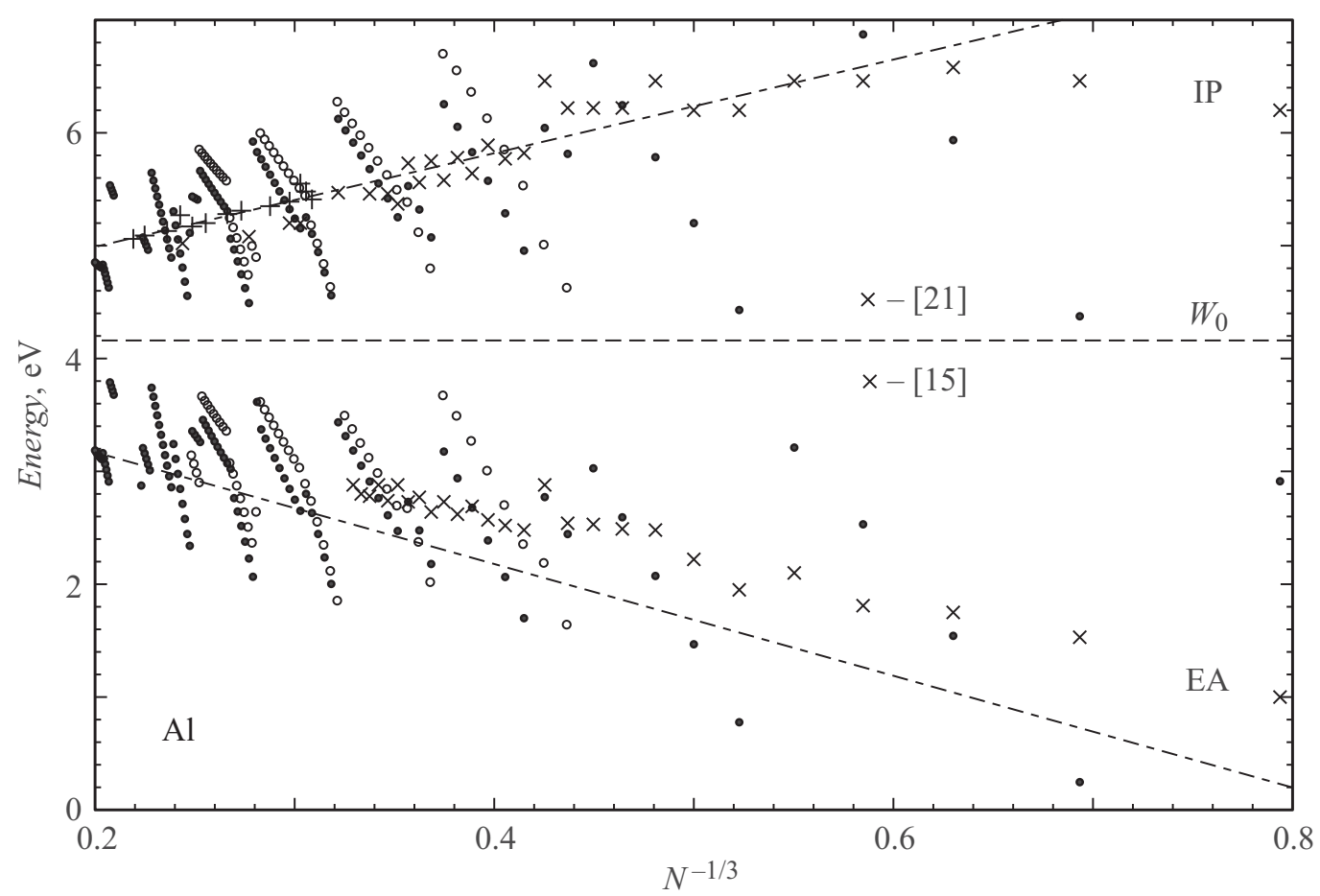

Рис. 3. Потенциал ионизации IP и энергия прилипания ЕА, вычисленные непосредственно по формулам (10) для сплошных кластеров (точки) и кластеров с моновакансией (пустые кружки); экспериментальные значения (кресты) для $\mathrm{Al}$ [15,21]. Штрихпунктирная линия - асимптотика (12).

различие наблюдается при переходе с полностью заполненной оболочки на пустую. По мере увеличения $N$, это различие нивелируется.

Результаты вычислений на рис. 3 для $\mathrm{Al}$ приведены в координатах $N^{-1 / 3}$ как для сплошных кластеров, так и для кластеров, содержащих моновакансию. Для кластеров с моновакансией $c_{v}=1 / N$, поэтому имеется соответствие $N^{-1 / 3}=c_{v}^{1 / 3}$. В нашем случае $c_{v} \rightarrow 0$ при $N \rightarrow \infty$. Если вакансия не одна, но их концентрация мала (вакансии не взаимодействуют друг с другом), по нашим рисункам возможно качественно отслеживать зависимость энергетических характеристик от концентрации вакансий.

На рис. 3 приведены также асимптотики величин IP и $E A$ :

$$
\begin{aligned}
& I P_{N}=-\mu_{0}+\alpha e^{2} / R_{N}, \\
& E A_{N}=-\mu_{0}-\beta e^{2} / R_{N},
\end{aligned}
$$

записанные в соответствии с разложением химического потенциала электронов по степеням обратного радиуса нейтрального кластера

$$
\mu\left(R_{N}\right)=\mu_{0}+\mu_{1} / R_{N}+O\left(R_{N}^{-2}\right) .
$$

Здесь $\mu_{0}=-W_{0}, W_{0}-$ работа выхода металла при $R_{N} \rightarrow \infty$. Коэффициенты $\alpha=1 / 2-\mu_{1} / e^{2}$ и $\beta=$ $=1 / 2+\mu_{1} / e^{2}$ содержат параметр $\mu_{1}=2 \sigma_{0} / \bar{n}$, характерный для каждого материала; $\sigma_{0}-$ удельная энергия плоской поверхности (предел $N \rightarrow \infty$ ).
Потенциал ионизации и энергия прилипания демонстрируют сильное осцилляционное поведение, обусловленное сферической оболочечной структурой. Они стремятся к $W_{0}$ асимптотически достаточно медленно, что обусловлено высоким орбитальным вырождением и большими угловыми квантовыми числами $l$. По результатам экспериментов осцилляции гораздо слабее. Выход за рамки LDA и использование local spin density approximation (LSDA) позволяют уменьшить осцилляции.

Используя теорему Купменса, формулы (10) можно переписать в виде

$$
\begin{aligned}
& I P_{N, v}=\varepsilon_{N, v}^{\mathrm{HO}}+\frac{e^{2}}{2 C_{N, v}^{+}}, \\
& E A_{N, v}=\varepsilon_{N, v}^{\mathrm{LU}}-\frac{e^{2}}{2 C_{N, v}^{-}},
\end{aligned}
$$

где $\varepsilon_{N, v}^{\mathrm{HO}} / \varepsilon_{N, v}^{\mathrm{LU}}$ и $C_{N, v}^{+}-$энергии верхних занятых/нижних незанятых электронных орбиталей кластера и электрические емкости соответственно.

На рис. 4 приведены спектры для сплошных кластеров $\mathrm{Al}$ и Na. Рис. 4 демонстрирует заполнение электронных оболочек по мере увеличения числа электронов. Сплошными черточками обозначены заполненные, а пунктирными - незаполненные (виртуальные) электронные уровни. Также на рисунке помечены верхний занятый $\varepsilon_{N}^{\mathrm{HO}}$ (точка на сплошной линии) и нижний не занятый $\varepsilon_{N}^{\mathrm{LU}}$ (кружок) энергетические уровни. 

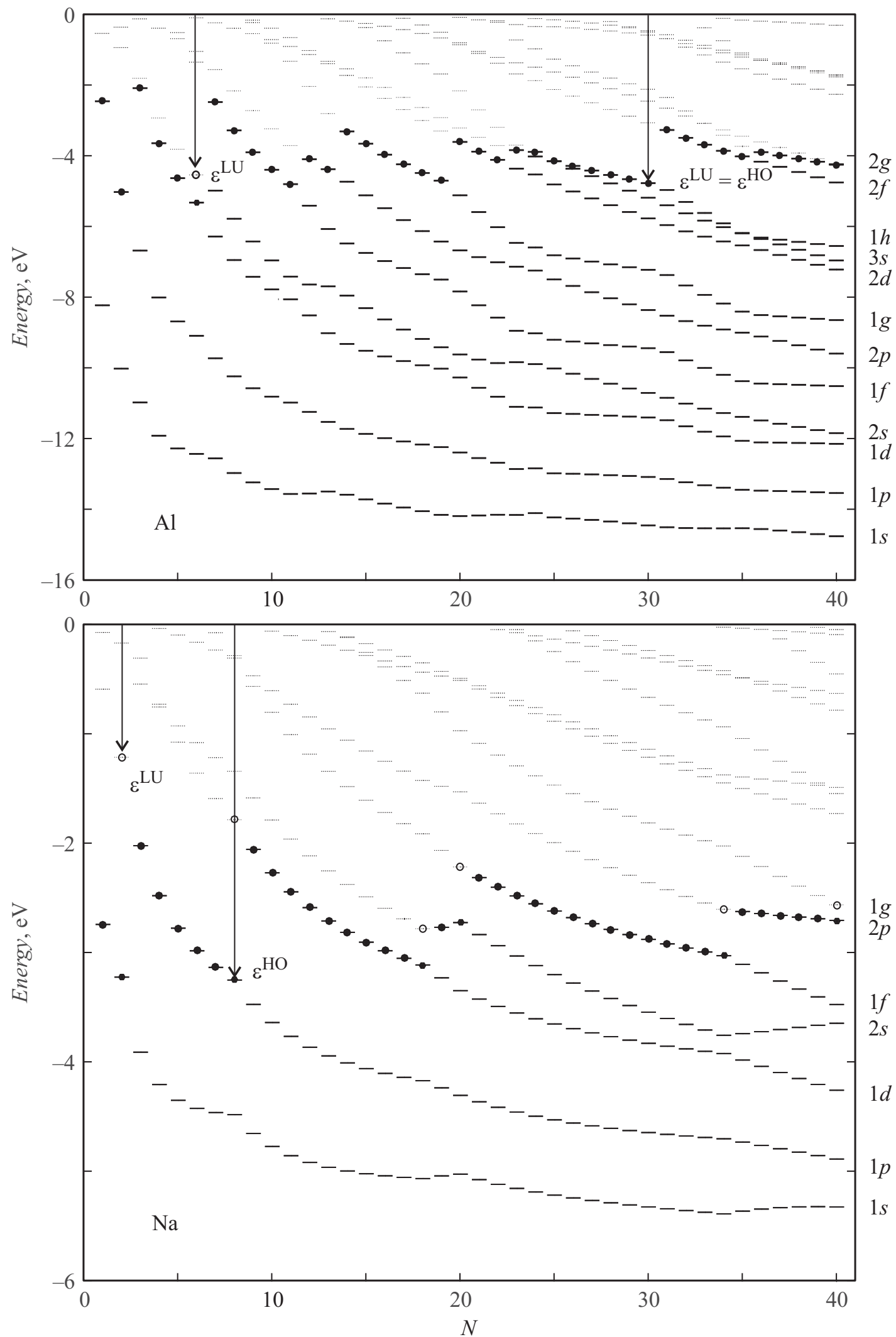

Рис. 4. Спектры сплошных кластеров $\mathrm{Al}$ и $\mathrm{Na}$.

Для частично заполненных оболочек $\varepsilon_{N, v}^{\mathrm{HO}}=\varepsilon_{N, v}^{\mathrm{LU}} \approx$ $\approx \mu\left(R_{N, v}\right)$. Максимальные значения $\varepsilon_{N, v}^{\mathrm{HO}}$ соответствуют полностью заполненным оболочкам, а магические числа атомов $N^{* *}$ для сферических сплошных кластеров и 


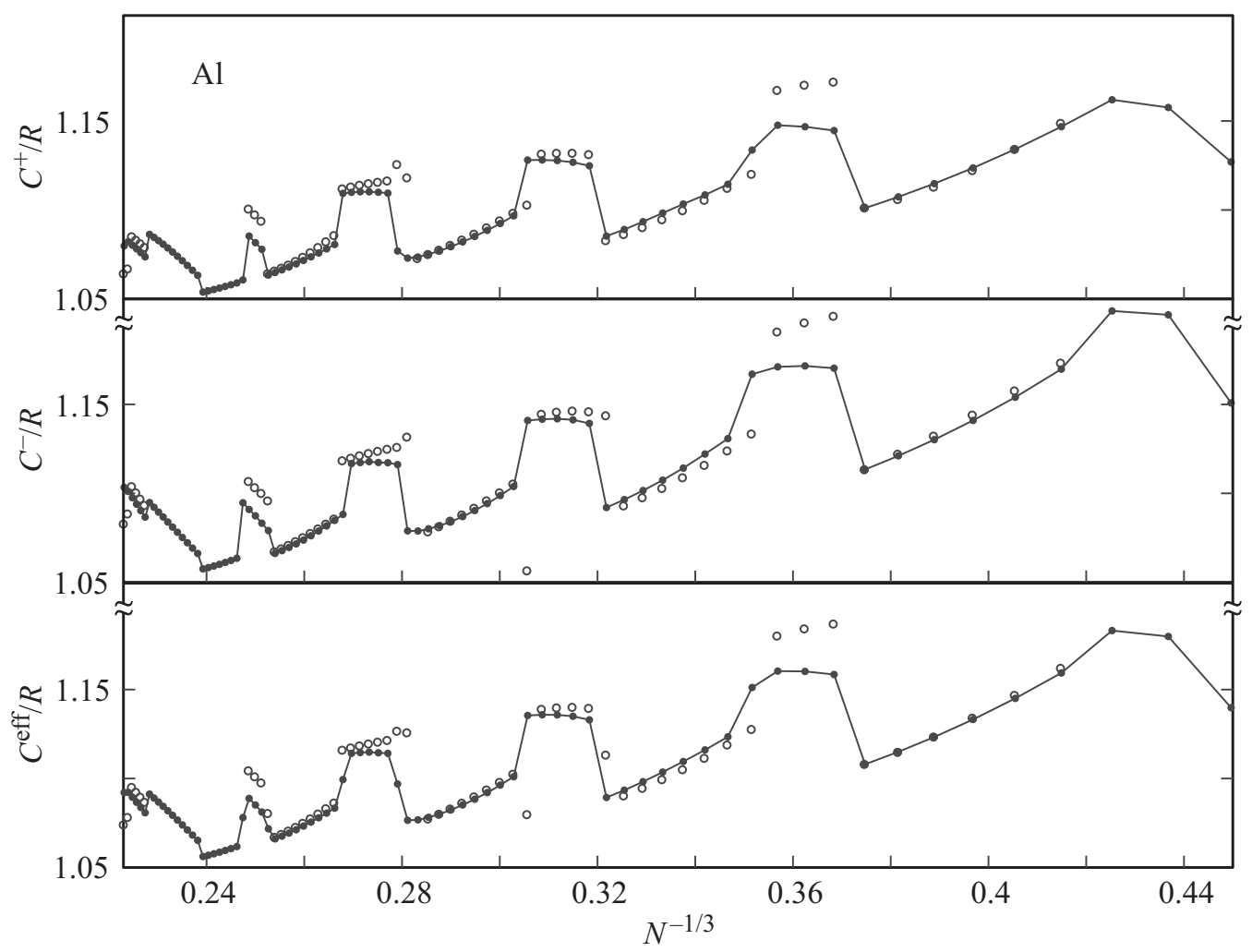

Рис. 5. Результаты расчета приведенных емкостей сплошных кластеров Al (точки соединены линиями) и кластеров с моновакансией (кружки).

кластеров с вакансией не во всех случаях совпадают. Для $\mathrm{Na}$ получены значения $N^{* *}=2,8,18,20,34,40$, $58,68,90,92,106,132,138,168,186,196,198,(230)$, $232,(252), 254$. Для поливалентного $\mathrm{Al} N^{* *}=6,(30)$, $44,46,52,62,\{66\},(84),(102),\{104\},\{136\}, 146$, $\{154\},(180),(202), 204$. В круглых скобках приведены те значения для дефектных кластеров, которые не совпадают с соответствующими для сплошных кластеров, а в фигурных - наоборот.

С увеличением $R_{N, v}$ величины $-\varepsilon_{N, v}^{\mathrm{HO}}$ и $-\varepsilon_{N, v}^{\mathrm{LU}}$, осциллируют и стремятся к $-\mu\left(R_{N, v}\right)$ при $R \rightarrow \infty$. Амплитуда осцилляций убывает примерно как $1 / R_{N, v}^{3}$.

Вернемся к рис. 2 и 3. Обозначим $\Delta\left(I P_{N}\right)=I P_{N}-$ $-I P_{N, v}$. На первый взгляд неожиданным является знак $\Delta\left(I P_{N}\right)<0$ (кружки располагаются выше точек при одном и том же $N)$. Исключение составляют кластеры с такими $N$, при которых максимальный вклад дают уровни с низкими $l(s, p$ - и частично $d$-орбитали). На рис. 2 эти узкие области заключены между вертикальными шрихпунктирными линиями.

Из анализа асимптотического поведения $I P_{n}$ и $I P_{N, v}$ следует, что $\Delta\left(I P_{N}\right)>0$. Основная вакансионная зависимость содержится в работе выхода $W_{0}\left(c_{v}\right)<$ $<W_{0}\left(c_{v}\right)=0[22]$.

В случае малых кластеров с моновакансией существенным становится возмущение от вакансии, концентрация которой $c_{v} \sim 1 / R_{N}^{3}$. Как следует из рис. 1 , поведение $v_{\text {effv }}(r)$ таково, что электроны выдавливаются вакансией из центра кластера к поверхности и группируются, главным образом, в шаровом слое $r_{0}<r<R_{N}$. А при интегрировании (3) в сферических координатах именно эта область дает основной вклад в энергию. Это подтверждается спектральными значениями энергий, соответствующих точкам (кружкам) для $\mathrm{Na}$ на рис. 2. Например, для $N=12 \quad \varepsilon_{n_{r=0, l=0}}=-4.925(-4.577) \mathrm{eV}$, $\varepsilon_{n_{r-0, H}}=-3.871(-3.831) \mathrm{eV}, \varepsilon_{n_{r=0, t 2}}^{\mathrm{HO}, \mathrm{LU}}=-2.595(-2.708) \mathrm{eV}$, а также для $N=18 \varepsilon_{n_{\vdash \rightarrow 0, H 0}}=-5.073(-4.755) \mathrm{eV}, \varepsilon_{n_{t \rightarrow 0, H}}=$ $=-4.177(-4.135) \mathrm{eV}, \quad \varepsilon_{n_{r=0, l=2}}^{\mathrm{HO}}=-3.119(-3.189) \mathrm{eV}$, $\varepsilon_{n_{r=1, l=0}}^{\mathrm{LU}}=-2.787(-2.048) \mathrm{eV}$.

C ростом $N$ вклад от объема кластера становится все существенней и в асимптотике точки и кружки поменяются местами, т.е. разность $\Delta\left(I P_{N \rightarrow \infty}\right)$ становится положительной по знаку.

Вычисленные по общим формулам (10) самосогласованные значения $I P, E A, \varepsilon^{\mathrm{HO}}$ и $\varepsilon^{\mathrm{LU}}$, позволяют нам, воспользовавшись выражениями (10), рассчитать емкости

$$
\begin{gathered}
C_{N, v}^{+}=\frac{e^{2}}{2\left(I P_{N, v}+\varepsilon_{N, v}^{\mathrm{HO}}\right)}, \\
C_{N, v}^{-}=\frac{-e^{2}}{2\left(E A_{N, v}+\varepsilon_{N, v}^{\mathrm{LU}}\right)}, \\
C_{N, v}^{\mathrm{eff}}=\frac{e^{2}}{2\left(I P_{N, v}+\varepsilon_{N, v}^{\mathrm{HO}}-E A_{N, v}-\varepsilon_{N, v}^{\mathrm{LU}}\right)} .
\end{gathered}
$$


Аналогичные формулы для $C_{N}$ соответствуют и бездефектным кластерам.

В классической электростатике емкости проводящих шаров определяются их внешними радиусами $R_{N, v}$. Шероховатость поверхности на атомном масштабе (атомы имеют конечный объем) не позволяет точно установить границу [23]. В модели желе границе ионного остова всегда отвечает координата $r=R_{N, v}$. Однако, электронное облако все более и более „выплескивается“ за границу остова по мере уменьшения его радиуса $R_{N, v}$. Более того, такое „выплескивание“ зависит от знака избыточного заряда кластера (рис. 1). В связи с этим величины $C_{N, v}^{\mathrm{eff}}, C_{N, v}^{+}$и $C_{N, v}^{-}$равны друг другу только в асимптотике при $N \rightarrow \infty$.

На рис. 5 для $\mathrm{Al}$ представлены результаты вычислений емкостей $C_{N}$ и $C_{N, v}$, нормированных на свой радиус $R_{N}$ и $R_{N, v}$ (атомные единицы) соответственно. Наибольшее различие наблюдается для интервалов $N$, в которых происходит заполнение $s$ - и $p$-электронных оболочек. Знакопеременная разность

$$
C_{N, v} / R_{N, v}-C_{N} / V_{N}
$$

определяется, главным образом, соотношением величин $\varepsilon^{\mathrm{HO}}$ и $\varepsilon^{\mathrm{LU}}$ для разных $l$ в сплошных и дефектных кластерах, которое может меняться в зависимости от главного квантового числа. Емкость дефектных кластеров при заполнении оболочек с малым $l$ больше чем у сплошных, а для больших $l$ наблюдается обратное соотношение. Для $\mathrm{Na}$ нормированные величины $C_{N}$ и $C_{N, v}$ принципиально не отличаются от приведенных на рис. 5.

Используя экспериментальные данные для $N=1$ (атома) $\mathrm{Na}\left(I P_{1}=5.14 \mathrm{eV}, E A_{1}=0.55 \mathrm{eV}, R_{1}=r_{0}\right)$, а также условие $\varepsilon_{1}^{\mathrm{HO}}=\varepsilon_{1}^{\mathrm{LU}}$ для незаполненных оболочек, в качестве теста получим величину $C_{1}^{\mathrm{eff}} / r_{0}=1.8$. Это значение неплохо согласуется с рассчитанными величинами, представленными на рис. 5 для самых маленьких кластеров. Для незамкнутых электронных оболочек кластер может иметь более низкую симметрию, например сфероидальную.

\section{Энергии диссоциации, когезии и образования вакансии}

Энергия диссоциации нейтрального металлического $(\mathrm{Me})$ кластера в соответствии с реакцией $\mathrm{Me}_{N} \rightarrow \mathrm{Me}_{N-1}+\mathrm{Me}_{\text {at }}$ определяется разницей полных энергий

$$
\varepsilon_{N}^{\mathrm{dis}}=\left[E_{N-1}+E_{\mathrm{at}}\right]-E_{N}=N \varepsilon_{N}^{\mathrm{coh}}-(N-1) \varepsilon_{N-1}^{\mathrm{coh}} .
$$

В модели стабильного желе энергия атома $E_{\text {at }}-$ это полная энергия металлической сферы радиуса $r_{0}$.

Измерения энергии диссоциации и потенциала ионизации кластерных ионов металлов проводились неоднократно и детально комментировались $[15,20,21,24,25]$.
Традиционно по этим данным рассчитывается энергия когезии нейтральных кластеров.

По определению, энергия когезии $\varepsilon_{N}^{\mathrm{coh}}-$ это энергия связи (атомов в кластере), приходящаяся на один атом. Она определяется разностью совокупной энергии $N$ свободных атомов и энергии кластера, состоящего из $N$ атомов:

$$
\varepsilon_{N}^{\mathrm{coh}}=\left(N E_{\mathrm{at}}-E_{N}\right) / N=E_{\mathrm{at}}-E_{N} / N .
$$

При $N \rightarrow \infty \varepsilon_{N}^{\mathrm{coh}} \rightarrow \varepsilon_{\infty}^{\mathrm{coh}} \equiv \varepsilon^{\mathrm{coh}}\left(r_{0}\right)$. Вычисленные нами значения $\varepsilon^{\mathrm{coh}}\left(r_{0}\right)=3.97 \mathrm{eV}$ и $1.16 \mathrm{eV}$ соответственно для $\mathrm{Al}$ и $\mathrm{Na}$ неплохо согласуются с экспериментальными значениями $\varepsilon_{\infty}^{\mathrm{coh}}=3.39$ и $1.11 \mathrm{eV}$ (см. работу [26] и ссылки в ней).

Уравнение связи имеет вид

$$
\varepsilon_{N}^{\mathrm{coh}}=\frac{1}{N} \sum_{n=2}^{N} \varepsilon_{n}^{\mathrm{dis}} .
$$

Асимптотика размерной зависимости энергии когезии (15) представляет собой известный классический результат [1]

$$
\varepsilon_{N}^{\mathrm{coh}}=\varepsilon^{\mathrm{coh}}\left(r_{0}\right)-\frac{2 \sigma_{0}}{n_{\mathrm{at}} R_{N}},
$$

где последнее слагаемое может быть записано как $-Z \mu_{1} / R_{N}$.

Следует отметить, что еще в работах Френкеля и Ленгмюра было замечено, что для некоторых веществ при низких температурах соблюдается универсальное соотношение

$$
4 \pi r_{0}^{2} \sigma / q \approx 2 / 3,
$$

составленное из наблюдаемых величин: среднего расстояния между атомами $r_{0}$, удельной поверхностной энергии $\sigma$ и теплоты испарения $q$ (см. таблицу в [23]). Используя это соотношение, асимптотику (17) можно переписать в виде, удобном для оценок:

$$
\varepsilon_{N}^{\mathrm{coh}} \approx \varepsilon^{\mathrm{coh}}\left(r_{0}\right)\left[1-(4 / 9) N^{-1 / 3}\right] .
$$

Далее, используя формулу (17) и определение (14), обнаруживается совпадение асимптотик $\varepsilon_{N}^{\text {coh }}$ и $\varepsilon_{N}^{\text {dis }}$

$$
\varepsilon_{N}^{\mathrm{dis}}=\varepsilon^{\mathrm{coh}}\left(r_{0}\right)-\frac{2 \sigma_{0}}{n_{\mathrm{at}} R_{N}} .
$$

На рис. 6 приведены энергия диссоциации и когезии сплошных кластеров и кластеров, содержащих моновакансию Al. Размерная зависимость энергии диссоциации на рис. $6, a$ состоит из квантовых осцилляций вокруг своей асимптотики. У дефектного кластера при больших $l$ значения $\varepsilon_{N, v}^{\text {dis }}$ располагаются выше, чем у сплошного кластера, а для малых $l$ они меняются местами. Для сплошных и дефектных кластеров помимо смены порядка заполнения электронных уровней следует отметить также существенное различие в поведении энергии диссоциации с одинаковым числом атомов $N$ : для малых $l$ 


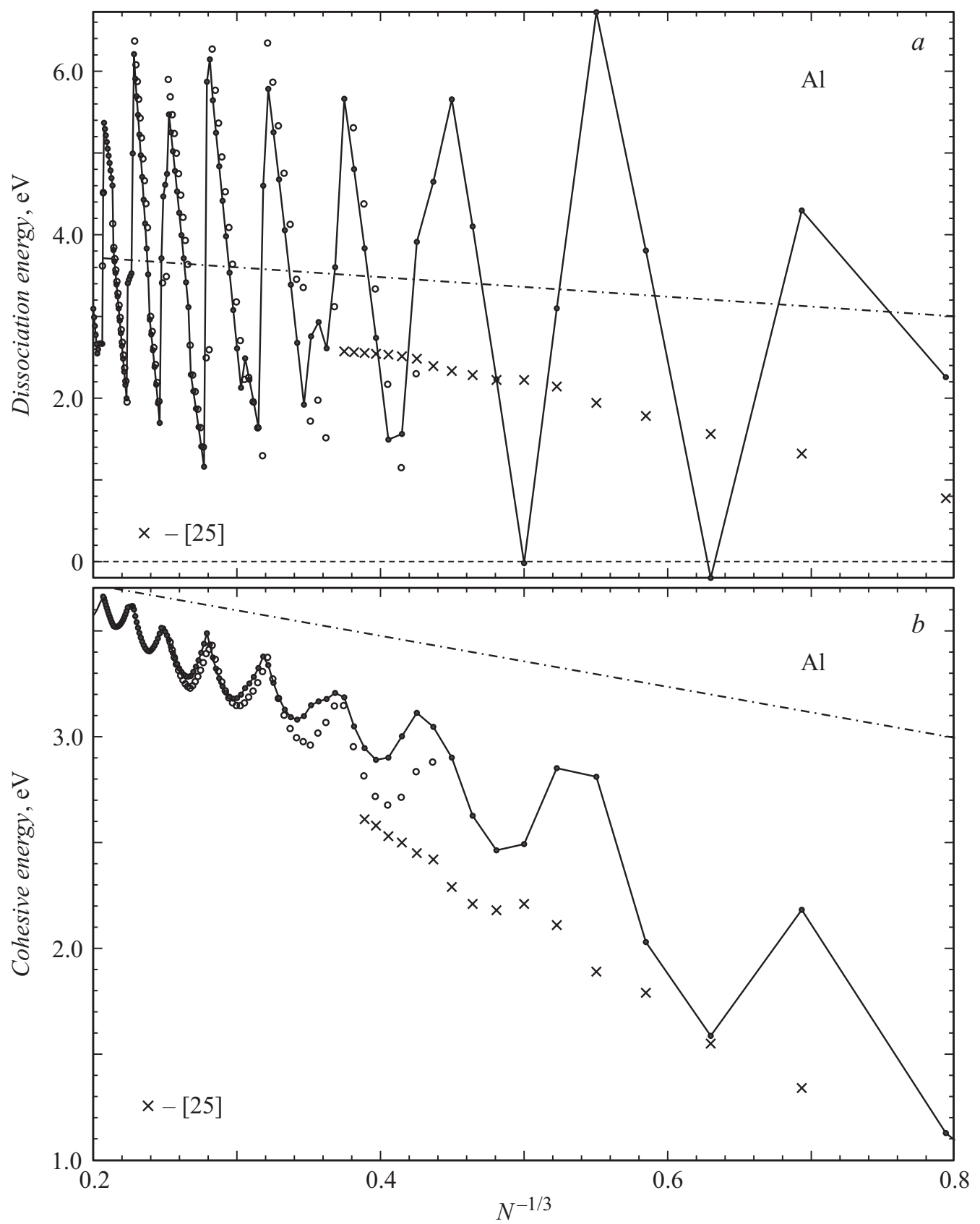

Рис. 6. Расчетные значения энергии диссоциации $\varepsilon^{\mathrm{dis}}(a)$ и энергии когезии $\varepsilon^{\mathrm{coh}}(b)$ : сплошные кластеры (точки соединены линиями), дефектные кластеры (кружки); экспериментальные значения [25] (кресты); штрихпунктирные линии - асимптотики (17) и (18).

энергия диссоциации сплошных кластеров уменьшается с ростом $N$, а дефектных - увеличивается. Сравнение данных рис. $6, a$ и $b$ подтверждает точность формулы (16), а также объясняет отличие в расположении локальных максимумов на рис. $6, a$ и $b$. Расчетные значения $\varepsilon_{N, v}^{\mathrm{coh}}$ располагаются ближе к экспериментальным значениям, полученным при $T=150 \mathrm{~K}[25]$, чем $\varepsilon_{N}^{\text {coh }}$. Отметим, что вблизи фазового перехода величины $\varepsilon_{N}^{\mathrm{dis}}$, выделенные из измерений температуры плавления и скрытой теплоты перехода могут быть отрицательными по знаку [10].
Таким образом, можно сделать вывод, что наиболее стабильными бездефектными кластерами являются те, у которых последними заполненными являются уровни с малым $l$, а для дефектных - наоборот. В экспериментах размерные осцилляции $\varepsilon_{N}^{\text {dis }}$, по-видимому, подавлены температурными эффектами (см. рис. 9 в [24]).

Расчету из первых принципов (ab initio) энергии образования вакансии в металлах посвящено значительное количество работ [27]. В модели стабильного желе и liquid drop model энергия когезии атома и энергия образования вакансии исследованы в виде Padé- 

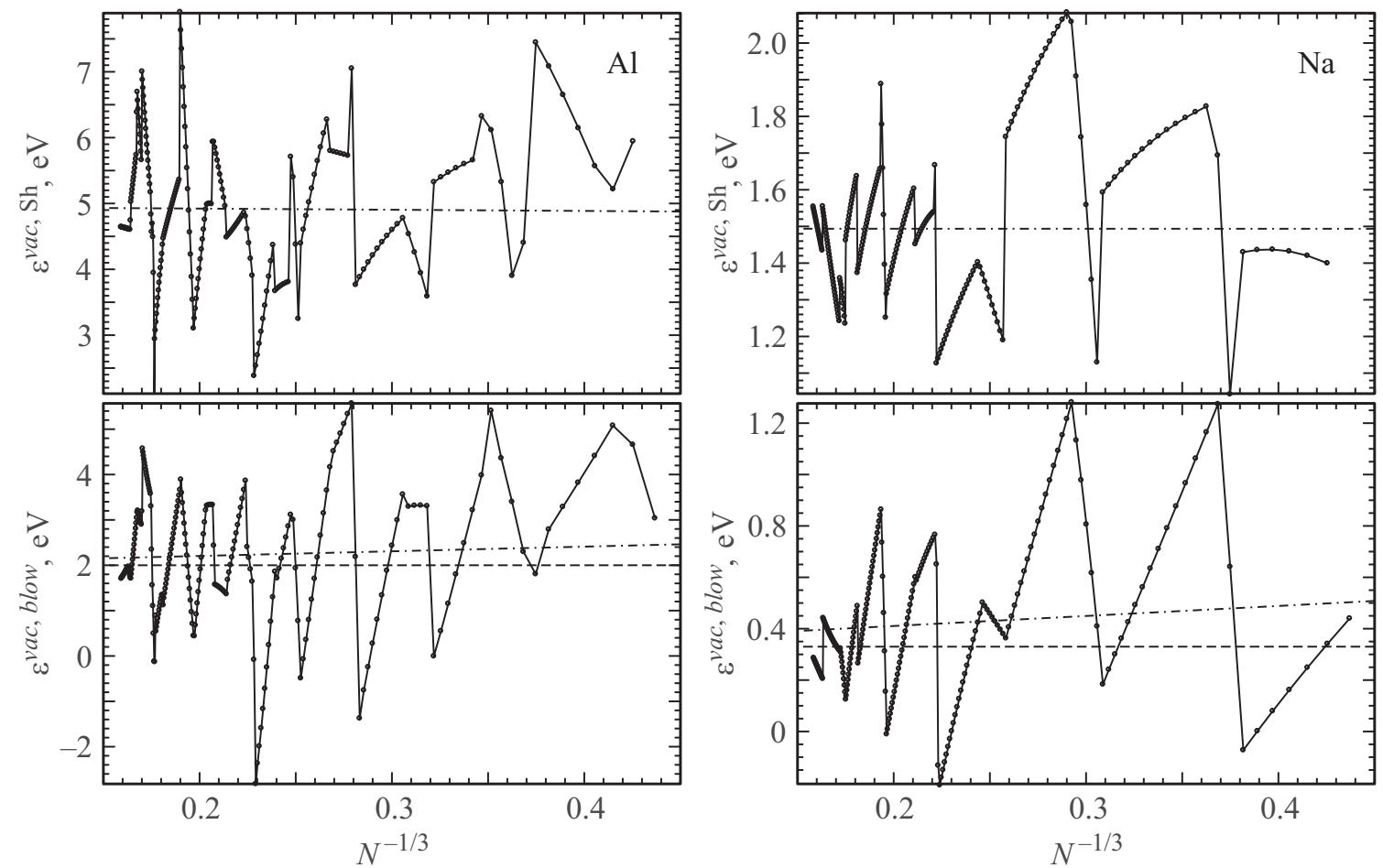

Рис. 7. Расчетные значения энергии образования моновакансии по Шоттки $\varepsilon_{N, v}^{\mathrm{vacSh}}(21)$ и по механизму выдувания пузырька $(22)$ для $\mathrm{Al}$ и Na. Штрихпунктирные линии - асимптотики (24) и (23). Горизонтальные штриховые линии $-\varepsilon_{\infty}^{\mathrm{vac}} \equiv \varepsilon^{\text {vac }}\left(r_{0}\right)=1.0$ и $0.33 \mathrm{eV}$ для $\mathrm{Al}$ и $\mathrm{Na}$ соответственно.

разложения [26] (см. также [23]). В обозначениях работы [23] результаты [26] выглядят следующим образом:

$$
\begin{aligned}
& \varepsilon^{\mathrm{coh}}\left(r_{0}\right)=4 \pi r_{0}^{2} \sigma_{0}\left(1+\delta_{1} / r_{0}+\delta_{2} / r_{0}^{2}\right), \\
& \varepsilon^{\mathrm{vac}}\left(r_{0}\right)=4 \pi r_{0}^{2} \sigma_{0}\left(1-\delta_{1} / r_{0}+\delta_{2} / r_{0}^{2}\right) .
\end{aligned}
$$

Вычисленные недавно в [28] с помощью фаз рассеяния электронных волн на вакансионном потенциале значения $\varepsilon_{\infty}^{\mathrm{vac}} \equiv \varepsilon^{\mathrm{vac}}=1.00$ и $0.33 \mathrm{eV}$ соответственно для $\mathrm{Al}$ и $\mathrm{Na}$ согласуются с экспериментальными значениями 0.73 и $0.335 \mathrm{eV}[26]$.

Используя величины $\varepsilon^{\mathrm{coh}}\left(r_{0}\right)$ и $\varepsilon^{\mathrm{vac}}\left(r_{0}\right)$, а также $\delta_{2} / r_{0}^{2}=-0.13(\mathrm{Na}),+0.22(\mathrm{Al})$ из [26], находим $\delta_{1} / r_{0}=0.32(\mathrm{Na}), 0.57(\mathrm{Al})$. Величины $\delta_{1}$ и $\delta_{2}$ нам необходимы для построения асимптотики энергии образования вакансии.

Для кластеров самосогласованные вычисления $\varepsilon_{N, v}^{\mathrm{vac}}$ не проводились вследствие необходимости детализации процесса образования вакансии. Поэтому представляет интерес выяснение выгодности образования вакансии по двум механизмам.

Механизм Шоттки: с поверхности сплошной сферы извлекается атом, и в конечном состоянии вакансия оказывается в центре сферы:

$$
\varepsilon_{N, v}^{\mathrm{var}, \mathrm{Sh}}=\left[E_{N-1, v}+E_{\mathrm{at}}\right]-E_{N}=N \varepsilon_{N}^{\mathrm{coh}}-(N-1) \varepsilon_{N-1, v}^{\mathrm{coh}},
$$

где $E_{N-1, v}-$ энергия сферы, содержащей в центре вакансию радиусом $r_{0}$ (шаровой слой между $r=r_{0}$ и $r=R_{N-1}$ содержит $N-1$ атомов).
Суть другого механизма [23], при котором число атомов в сфере не меняется, в „выдувании“дырки-вакансии радиуса $r_{0}$ в центре сферы:

$$
\varepsilon_{N, v}^{\mathrm{vac}, \mathrm{blow}}=E_{N, v}-E_{N}=N\left(\varepsilon_{N}^{\mathrm{coh}}-\varepsilon_{N, v}^{\mathrm{coh}}\right) .
$$

Сравнение (21) и (22) демонстрирует выгодность второго механизма соотношением

$$
\varepsilon_{N, v}^{\mathrm{vac}, \mathrm{sh}}=\varepsilon_{N, v}^{\mathrm{vac}, \text { blow }}+\varepsilon_{N, v}^{\mathrm{dis}} .
$$

Исследуем асимптотику энергии образования вакансии. Ее размерная зависимость определяется разностью полных энергий сфер, рассчитанных по формулам (21) и (22) в пределе $N \rightarrow \infty$, и сводится к разности полных поверхностных энергий.

Для механизма выдувания вакансии, используя (10), полагая $R_{N, v}=R_{N}\left(1+\frac{1}{3} \xi^{3}\right)$, где $\xi \equiv r_{0} / R_{N} \ll 1$, и удерживая нужный порядок разложения, получим

$$
\begin{aligned}
& \varepsilon_{N, v}^{\mathrm{vac}, \text { blow }}=4 \pi R_{N, v}^{2} \sigma_{0}\left(1+\delta_{1} / R_{N, v}+\delta_{2} / R_{N, v}^{2}\right) \\
& +\varepsilon^{\mathrm{vac}}\left(r_{0}\right)-4 \pi R_{N}^{2} \sigma_{0}\left(1+\delta_{1} / R_{N}+\delta_{2} / R_{N}^{2}\right) \\
& =\varepsilon^{\mathrm{vac}}\left(r_{0}\right)\left(1+\frac{2}{3 N^{1 / 3}\left(1-\delta_{1} / r_{0}+\delta_{2} / r_{0}^{2}\right)}\right) .
\end{aligned}
$$

Для механизма Шоттки в соответствии с (23) и $R_{N-1, v}=R_{N}$ асимптотика определяется суммой выражений (24) и (18). Асимптотическая зависимость $\varepsilon_{N, v}^{\mathrm{vac}, \mathrm{Sh}}$ 
слабо зависит от $N$, а зависимость (24) демонстрирует уменьшение энергии образования вакансии с ростом $N$, что согласуется с выводами работы [29] и противоречит [12-14].

На рис. 7 приведены результаты вычислений энергии образования вакансий по двум механизмам. Эти вычисления подтверждают формулу (23), а именно выгодность выдувания вакансии. Все зависимости сильно осциллируют. Для некоторых $N$, особенно для $\mathrm{Al}$, значения $\varepsilon_{N, v}^{\text {vac,blow }}$ становятся отрицательными в узких диапазонах $N$. Именно такие участки обозначены на рис. 2 и выше в тексте уже даны комментарии по иерархии электронных состояний в таких кластерах.

Приведенные вычисления в LDA соответствуют нулевой температуре. Возможно, при плотности атомов, соответствующих конечным температурам, пониженной симметрии кластеров, а также использованию LSDA для обменно-корреляционной энергии сильное осцилляционное поведение энергетических характеристик будет подавлено. Во всяком случае, размерное поведение результатов прямых вычислений хорошо согласуется со своими асимптотиками.

В квазитермодинамике выгодность появления вакансий в кластере при конечной температуре $T$ может быть оценена из условия изменения свободной энергии $\Delta F_{N, v}^{\text {vac,blow }}$ системы

$$
\Delta F_{N, v}^{\text {vac,blow }}=\varepsilon_{N, v}^{\text {var,blow }}-T \Delta S_{N, v}^{\text {vac,blow }}=\leq 0 .
$$

Вследствие того, что при выдувании вакансии число ионов в кластере не меняется, энтропийный вклад формируется только вырожденным электронным газом. Соответствующее выражение имеет вид

$$
\begin{aligned}
T \Delta S_{N, v}^{\mathrm{vac}, \text { blow }} & =\frac{2 \pi^{5 / 3}}{3^{2 / 3}}\left(\frac{k_{B} T}{e^{2}}\right)^{2} \\
\times & \int_{0}^{\infty} d r r^{2}\left[n_{N, v}^{1 / 3}(r)-n_{N}^{1 / 3}(r)\right] .
\end{aligned}
$$

Для вычислений в (26) потребуются равновесные профили распределений электронов в модели стабильного желе при заданных $N$ и $T$. При нулевой температуре и $N=12$ эти профили представлены на рис. 1 .

\section{Заключение}

Методом Кона-Шема в модели стабильного желе выполнены самосогласованные вычисления профилей радиальных распределений электронов и потенциалов сплошных кластеров и кластеров с центрированной моновакансией. Это позволило впервые определить полную энергию нейтрального и заряженного дефектного кластера, на основе чего проведены прямые вычисления энергий диссоциации, когезии, образования вакансии, прилипания электронов и потенциала ионизации, а также электрической емкости. В качестве примера были выбраны простые металлы Al и Na. Результаты вычислений сопоставлены с асимптотиками и результатами для бездефектных кластеров. Компьютерный код вычислений авторами составлен самостоятельно.

Потенциал ионизации для самого малого кластера с вакансией больше (примерно на $0.5 \mathrm{eV}$ для $\mathrm{Al}$ и на $0.1 \mathrm{eV}$ для $\mathrm{Na})$, чем для сплошного кластера. Максимальное различие наблюдается при переходе с полностью заполненной оболочки на пустую. По мере увеличения $N$ это различие исчезает. Магические числа атомов для сплошных кластеров и кластеров с вакансией отличаются, особенно для Al.

Нормированные электрические емкости кластеров всегда превышают единицу и содержат квантовые размерные флуктуации. При этом для дефектных кластеров с частично заполненными электронными оболочками емкость заметно больше, чем для сплошных кластеров.

Впервые рассчитаны квантово-размерные зависимости энергии образования вакансии по механизмам Шоттки и „выдувания пузырька“ и определены их асимптотические тенденции. Отмечены сильные размерные флуктуации во всей области размеров кластеров. Размерные асимптотики этих двух механизмов отличны друг от друга и слабо зависят от числа атомов в кластере.

Размерная зависимость энергии когезии содержит локальные максимумы. Кластеры, им соответствующие, являются более устойчивыми, т.е. имеют бо́льшие по величине энергии связи, диссоциации и образования вакансии, чем их соседи. Для маленьких кластеров такими максимумами с ростом $N$ завершается заполнение очередной электронной оболочки. Положение максимумов для дефектных и бездефектных кластеров различно, что обусловлено не только различием их размеров, но и характером поведения волновых функций электронов. С ростом $N$ на участках между максимумами энергия диссоциации либо постоянно возрастает, либо имеет локальный минимум, а энергия образования вакансий монотонно убывает.

Авторы выражают благодарность О.В. Василенко за чтение рукописи и критические замечания.

\section{Список литературы}

[1] Френкель Я.И. Кинетическая теория жидкостей. М.-Л.: Изд-во АН СССР, 1959. 460 с.

[2] Safaei A. // Phil. Mag. 2011. Vol. 91. N 10. P. 1509-1539.

[3] Luo W., Su K., Li K., Liao G., Hu N., Jia M. // J. Chem. Phys. 2012. Vol. 136. N 23. P. 234704.

[4] Chandra J., Kholiya K. // Mod. Phys. Lett. B. 2015. Vol. 29. N 8. P. 1550025.

[5] Булавин Л.А., Актан О.Ю., Забашта Ю.Ф. // ФТТ. 2010. Т. 52. Вып. 4. С. 662-667.

[6] Магомедов М.Н. // ЖТФ. 2016. Т. 86. Вып. 5. С. 92-95.

[7] $Q i W$.// Acc. Chem. Res. 2016. Vol. 49. N 9. P. 1587-1595.

[8] Breaux G.A., Neal C. M., Cao B., Jarrold M.F. // Phys. Rev. Lett. 2005. Vol. 94. N 17. P. 173401. 
[9] Hock C., Bartels C., Straßburg S., Schmidt M., Haberland H., von Issendorff B., Aguado A. // Phys. Rev. Lett. 2009. Vol. 102. N 4. P. 043401.

[10] Starace A.K., Cao B., Judd O.H., Bhattacharyya I., Jarrold M.F. // J. Chem. Phys. 2010. Vol. 132. N 3. P. 034302.

[11] Zamith S., Labastie P., Chirot F., L'Hermite J.-M. // J. Chem. Phys. 2010. Vol. 133. N 15. P. 154501.

[12] Yang C.C., Li S. // Phys. Rev. B, PRB. 2007. Vol. 75. N 16. P. 165413.

[13] Hendy S.C. // Nanotechnology. 2007. Vol. 18. N 17. P. 175703.

[14] Guisbiers G. // Nanoscale Res. Lett. 2010. Vol. 5. N 7. P. $1132-1136$.

[15] de Heer W.A. // Rev. Mod. Phys. 1993. Vol. 65. N 3. P. $611-676$.

[16] Vieira A., Torres M.B., Fiolhais C., Balbás L.C. // J. Phys. B. 1997. Vol. 30. N 15. P. 3583-3596.

[17] Aguado A., Lòpez J.M. // J. Chem. Phys. 2009. Vol. 130. N 12. P. 064704.

[18] Perdew J.P. // Prog. Surf. Sci. 1995. Vol. 48. P. 245-257.

[19] Babich A.V., Pogosov V.V. // Surf. Sci. 2010. Vol. 640. N 2. P. 210-216.

[20] Kostko $O$. Ph.D. dissertation. University of Freiburg, Germany, (2007), $195 \mathrm{p}$.

[21] Halder A., Kresin V.V. // J. Chem. Phys. 2015. Vol. 143. N 16. P. 164313.

[22] Бабич А.В., Погосов В.В., Рева В.И. // Письма в ЖТФ. 2016. Т. 42. Вып. 20. С. 11-17.

[23] Pogosov V.V. // Solid State Commun. 1994. Vol. 89. N 12. P. 1017-1021.

[24] Bréchignac C., Cahuzac Ph., Leygnier J., Weiner J. // J. Chem. Phys. 1989. Vol. 90. N 3. P. 1492-1498.

[25] Ray U., Jarrold M.F., Bower J.E., Kraus J.S. // J. Chem. Phys. 1989. Vol. 91. N 5. P. 2912-2921.

[26] Ziesche P., Perdew J.P., Fiolhais C. // Phys. Rev. B, PRB. 1994. Vol. 49. N 12. P. 7916-7928.

[27] Freysoldt C., Grabowski B., Hickel T., Neugebauer J., Kresse G., Janotti A., Van de Walle C.G. // Rev. Mod. Phys. 2014. Vol. 86. N 1. P. 253-305.

[28] Бабич А.В., Вакула П.В., Погосов В.В. // ФТТ. 2014. Т. 56. Вып. 5. С. 841-847.

[29] Delavari H., Madaah Hosseinia H.R., Simchia A. // Physica B. 2011. Vol. 406. N 20. P. 3777-3780. 